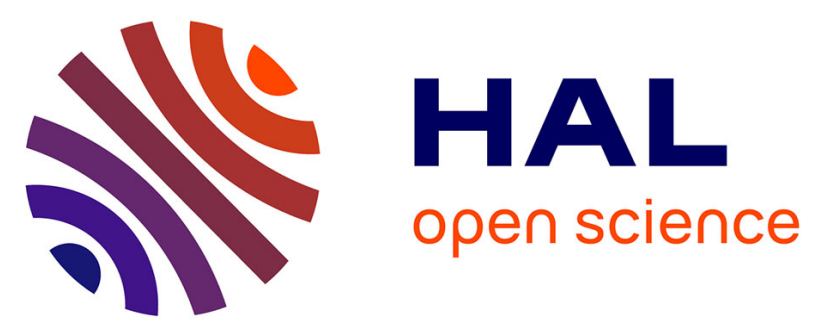

\title{
Analytic expressions of the magnetoresistance due to localization and electron-electron interaction effects. - Application to the amorphous alloys La3Al and La3Ga
}

\author{
J.C. Ousset, S. Askenazy, H. Rakoto, Jean-Marc Broto
}

\section{- To cite this version:}

J.C. Ousset, S. Askenazy, H. Rakoto, Jean-Marc Broto. Analytic expressions of the magnetoresistance due to localization and electron-electron interaction effects. - Application to the amorphous alloys La3Al and La3Ga. Journal de Physique, 1985, 46 (12), pp.2145-2149. 10.1051/jphys:0198500460120214500 . jpa-00210163

\section{HAL Id: jpa-00210163 https://hal.science/jpa-00210163}

Submitted on 1 Jan 1985

HAL is a multi-disciplinary open access archive for the deposit and dissemination of scientific research documents, whether they are published or not. The documents may come from teaching and research institutions in France or abroad, or from public or private research centers.
L'archive ouverte pluridisciplinaire HAL, est destinée au dépôt et à la diffusion de documents scientifiques de niveau recherche, publiés ou non, émanant des établissements d'enseignement et de recherche français ou étrangers, des laboratoires publics ou privés. 


\title{
Analytic expressions of the magnetoresistance due to localization and electron-electron interaction effects. Application to the amorphous alloys $\mathrm{La}_{3} \mathrm{Al}$ and $\mathrm{La}_{3} \mathrm{Ga}$
}

\author{
J. C. Ousset, S. Askenazy, H. Rakoto and J. M. Broto \\ Laboratoire de Physique des Solides, Service des Champs Intenses, I.N.S.A., Avenue de Rangueil, \\ 31077 Toulouse Cedex, France
}

(Reçu le 11 mars 1985, accepté sous forme définitive le 23 août 1985)

\begin{abstract}
Résumé. - Nous partons des calculs théoriques d'Alt'shuler et al. et de Lee et Ramakrishnan sur les contributions à la magnétorésistance de la localisation faible et des interactions électron-électron dans un système désordonné. Nous en donnons des formes analytiques d'utilisation facile pour les expérimentateurs. Nous présentons nos résultats expérimentaux sur les alliages amorphes $\mathrm{La}_{3} \mathrm{Al}$ et $\mathrm{La}_{3} \mathrm{Ga}$ et nous les interprétons à partir des équations que nous avons obtenues.

Abstract. - We start from the theoretical calculations of Alt'shuler et al. and Lee and Ramakrishnan for the contributions to magnetoresistance from weak localization and electron-electron interactions in a disordered system. We give analytic expressions which can be more easily handled by experimentalists. Our results on the amorphous alloys $\mathrm{La}_{3} \mathrm{Al}$ and $\mathrm{La}_{3} \mathrm{Ga}$ are presented and interpreted using these equations to get the best fit.
\end{abstract}

\section{Introduction.}

Weak localization effects in a disordered system were predicted by Anderson [1] in 1959. They have been widely studied during the last years in two-dimensional systems [2-4]. More recently they were observed in three-dimensional systems [5-7] and the amorphous metallic alloys [8-10] constitute an appropriate tool for these studies. Simultaneously theoretical models were developed and they allow the calculation of the magnetoresistance arising from weak localization [11, 12], superconducting fluctuations [13], and electronelectron interactions [12, 14]. Mathematical expressions have been established including functions of the magnetic field that are not easy to use by an experimenter who wants to interpret his results.

In section 2 we give the analytic forms of these expressions concerning three-dimensional systems, specifying the magnetic field range in which they constitute a very good approximation of the original equations. In order to illustrate these calculations we present, in section 3, the experimental results we have obtained by measuring the magnetoresistance of the amorphous metallic alloys $\mathrm{La}_{3} \mathrm{Al}$ and $\mathrm{La}_{3} \mathrm{Ga}$ containing praseodymium impurities. An important con- tribution from superconducting fluctuations and a strong spin-orbit scattering on lanthanum sites are observed.

In conclusion, we express some reserves concerning the validity of the theoretical models developed at time, in the presence of a high magnetic field. We shall discuss these problems in a further publication.

\section{Expressions of the magnetoresistance}

2.1 WeAK LOCALIZATION AND SUPERCONDUCTING FLUCTUATIONS. - The expression of the magnetoresistance due to weak localization and superconducting fluctuations (Maki-Thompson contribution) has been established by Alt'shuler et al. [12], Kawabata [11], and Larkin [13]. In the presence of strong spinorbit coupling $[12,8,15]$ the equation takes the following form :

$$
\begin{aligned}
\frac{\Delta \rho}{\rho}=\rho \frac{e^{2}}{2 \pi^{2} \hbar} & \left(\frac{e H}{\hbar}\right)^{1 / 2} \times \\
& \times\left\{\left(\frac{1}{2}+\beta\right) f_{3}\left(\frac{H}{H_{\mathrm{i}}}\right)-\frac{3}{2} f_{3}\left(\frac{H}{H_{\mathrm{so}}}\right)\right\}
\end{aligned}
$$


with :

$$
\begin{aligned}
H_{\mathrm{i}} & =\frac{\hbar}{4 e D} \tau_{\mathrm{i}}^{-1} \\
H_{\mathrm{so}} & =\frac{\hbar}{4 e D}\left(\tau_{\mathrm{i}}^{-1}+2 \tau_{\mathrm{so}}^{-1}\right)
\end{aligned}
$$

where $\tau_{\mathbf{i}}$ and $\tau_{\text {so }}$ are the electron relaxation times for inelastic and spin-orbit scattering, respectively. $D$ is the diffusion constant.

The term proportional to $\beta$ arises from the quenching of the superconducting fluctuations.

The function $f_{3}(h)$ is given by Kawabata [11]

$$
\begin{array}{r}
f_{3}(h)=\sum_{n=0}^{\infty} 2 \cdot\left\{\left(n+1+\frac{1}{h}\right)^{1 / 2}-\left(n+\frac{1}{h}\right)^{1 / 2}\right\} \\
-\frac{1}{\left(n+\frac{1}{2}+\frac{1}{h}\right)^{1 / 2}}
\end{array}
$$

$f_{3}$ is a slowly converging series.

We remark that :

$$
\begin{aligned}
f_{3}(h)=\lim _{N \rightarrow \infty}\{2(N+1 & \left.+\frac{1}{h}\right)^{1 / 2}-\frac{2}{\sqrt{h}} \\
& \left.-\sum_{n=0}^{N}\left(n+\frac{1}{2}+\frac{1}{h}\right)^{-1 / 2}\right\} .
\end{aligned}
$$

So using the Euler-Mac Laurin formula :

$$
\begin{aligned}
f_{3}=2 \cdot(s & \left.+\frac{1}{2}+\frac{1}{h}\right)^{1 / 2}-\frac{1}{2\left(s+\frac{1}{2}+\frac{1}{h}\right)^{1 / 2}}-\frac{2}{\sqrt{h}} \\
& -\sum_{n=0}^{s-1} \frac{1}{\left(n+\frac{1}{2}+\frac{1}{h}\right)^{1 / 2}+2} \\
& \times \sum_{p=1}^{\infty} B_{2 p}\left(\begin{array}{l}
1 / 2 \\
2 p
\end{array}\right) \frac{1}{\left(n+\frac{1}{2}+\frac{1}{h}\right)^{2 p-1 / 2}}
\end{aligned}
$$

for $s=2$, if limiting the series to $p=2, f_{3}$ is determined with an accuracy better than $5 \times 10^{-5}$ in all the magnetic field range :

$$
\begin{aligned}
f_{3}(h)=u-\frac{1}{u} & -\frac{1}{3 u^{3}}+\frac{1}{3 u^{7}}-\frac{2}{\sqrt{h}} \\
& -\left(\frac{1}{2}+\frac{1}{h}\right)^{-1 / 2}-\left(\frac{3}{2}+\frac{1}{h}\right)^{-3 / 2}
\end{aligned}
$$

with $u^{2}=10+4 / h$.

In the low and high-field limit, with an accuracy better than $10^{-3}$ we propose :

$$
f_{3}(h) \simeq \frac{h^{3 / 2}}{48}\left(1-\frac{7 h^{2}}{64}+\frac{91}{2048} h^{4}-\frac{3 h^{6}}{160}\right)
$$

for $h \leqslant 0.6$

$$
f_{3}(h) \simeq 0.6049-\frac{2}{h^{1 / 2}}+\frac{2.389}{h}-\frac{2.355}{h^{2}}
$$

for $h \geqslant 25$.

\section{2 ELECTRON-ELECTRON INTERACTION. ORBITAL PART.} - The magnetoresistance arising from electronelectron interaction has been calculated by Alt'shuler et al. [12]. It is worth noting that these authors only take into account the orbital part of this interaction. They obtain the following expression :

$$
\frac{\Delta \rho}{\rho}=\rho . g(T, H) \frac{e^{2}}{8 \pi^{2} \hbar}\left(\frac{e H}{\hbar}\right)^{1 / 2} \Phi_{3}\left(\frac{2 D e H}{\pi k T}\right) .
$$

The temperature dependence of the coupling constant $g$ was published by Larkin [13] a few years ago, and more recently its magnetic field dependence has been given by McLean and Tsuzuki [16] :

$$
g(T, H)^{-1}=\operatorname{Ln} \frac{T_{\mathrm{c}}}{T}+\Psi\left(\frac{1}{2}\right)-\Psi\left(\frac{1}{2}+\frac{D e H}{2 \pi k T}\right)
$$

where $\Psi(x)$ is the digamma function.

Writting $\left({ }^{1}\right)$

$$
\Psi(x)=-\gamma-\frac{1}{x}+\sum_{n=1}^{\infty}\left(\frac{1}{n}-\frac{x}{n+x}\right)
$$

and using the Euler-Mac Laurin formula, we propose :

$$
\begin{aligned}
& \Psi\left(\frac{1}{2}\right)-\Psi\left(\frac{1}{2}+x\right)= \\
& \quad-x \cdot \sum_{n=0}^{s-1} \frac{1}{(n+1 / 2)(n+1 / 2+x)} \\
& \quad-\operatorname{Ln}\left(1+\frac{x}{s+1 / 2}\right)-\frac{x}{2} \frac{1}{(s+1 / 2)(s+1 / 2+x)} \\
& \quad+\sum_{p=1}^{\infty} \frac{B_{2 p}}{2 p}\left\{\frac{1}{(s+1 / 2+x)^{2 p}}-\frac{1}{(s+1 / 2)^{2 p}}\right\} .
\end{aligned}
$$

For $s=2$, if limiting the series to the first term, the sum gives $\Psi(1 / 2)-\Psi(1 / 2+x)$ with an accuracy better than $7.3 \times 10^{-5}$ for any value of $x$. So for an easy computation we recommend the use of the formula :

$$
\begin{aligned}
& \Psi\left(\frac{1}{2}\right)-\Psi\left(\frac{1}{2}+x\right) \simeq \\
&-4 x\left\{\frac{1}{1+2 x}+\frac{1}{9+6 x}+\frac{85+32 x}{150(5+2 x)^{2}}\right\} \\
&-\operatorname{Ln}\left(1+\frac{2 x}{5}\right)
\end{aligned}
$$

(1) L. Schwartz, Meth. Math. pour les Sciences Physiques (Herman Editeur, Paris) p. 360. 
The function $\Phi_{3}(h)$ has the form :

$\Phi_{3}(h)=\left(\frac{\pi}{2 h}\right)^{1 / 2} \int_{0}^{\infty} \frac{t^{1 / 2}}{\sinh ^{2} t}\left\{1-\frac{h t}{\sinh h t}\right\} \mathrm{d} t$.

With an accuracy better than $2.5 \times 10^{-4}$, we propose for $\Phi_{3}$ three formulae to be used for the low field, for the high field, and for the medium range field, respectively.

For $h<1, \Phi_{3}$ is represented by the series

$$
\begin{aligned}
\Phi_{3}(h)=\sum_{n=1}^{\infty}( & -1)^{n} \cdot \pi \cdot(2 n+1)\left(1-2^{1-2 n}\right) \cdot B_{2 n} \\
& \times \xi_{2 n+1 / 2} \cdot\left(\begin{array}{c}
-1 / 2 \\
2 n+1
\end{array}\right) \cdot h^{2 n-1 / 2} .
\end{aligned}
$$

So limiting the formula to four terms, with the last one adapted to obtain the announced accuracy, for $h \leqslant 0.7$ :

$$
\begin{array}{r}
\Phi_{3}(h) \simeq 0.32925 h^{3 / 2}-0.11894 h^{7 / 2}+ \\
+0.10753 h^{11 / 2}-0.0636 h^{6.63}
\end{array}
$$

For $h \gg 1, \Phi_{3}$ is represented by a series of the form :

$$
\Phi_{3}(h)=\Phi_{3 \infty}-\frac{a_{0}}{\sqrt{h}}-\sum_{p=1}^{\infty}(-1)^{p} \frac{a_{p}}{h^{2 p}} .
$$

So limiting the formula to four terms and adjusting the last one, we recommend

$$
\begin{aligned}
\Phi_{3}(h) \simeq 1.900344- & 2.29392 / \sqrt{h} \\
+ & 1.2266 / h^{2}-0.826 / h^{3.5}
\end{aligned}
$$

for $h \geqslant 2.4$ :

$$
\begin{aligned}
\Phi_{3}(h) \simeq-0.03043 & +0.22616 h \\
& +0.14104 h^{2}-0.10293 h^{3} \\
& +0.02759 h^{4}-0.00028 h^{5} .
\end{aligned}
$$

For $0.7 \leqslant h \leqslant 2.4$

2.3 ElECtron-ElECTRON INTERACTION. SPIN EFFECTS. - The magnetoresistance of a weakly disordered electron gas, arising from spin-splitting of conduction electron energies, has been calculated by Lee and Ramakrishnan [14] in the case of a three-dimensional system :

$$
\frac{\Delta \rho}{\rho}=\rho \cdot \frac{e^{2}}{4 \pi^{2} h} \cdot F \cdot\left(\frac{k T}{2 \hbar D}\right)^{1 / 2} g_{3}\left(\frac{g \mu_{\mathrm{B}} H}{k T}\right)
$$

$F$ is the screening parameter for the Coulomb interaction and $g_{3}(h)$ is given by

$$
\begin{aligned}
g_{3}(h)=\int_{0}^{\infty} & \mathrm{d} \Omega \frac{\mathrm{d}^{2}}{\mathrm{~d} \Omega^{2}}\{\Omega N(\Omega)\} \\
& \times(\sqrt{\Omega+h}+\sqrt{|\Omega-h|}-2 \sqrt{\Omega})
\end{aligned}
$$

where

$$
N(\Omega)=\{\exp (\Omega)-1\}^{-1} .
$$

As for $\Phi_{3}$, we could not reach unique analytic form for $g_{3}$. First of all we remark that, for low $h, g_{3}$ is represented by a series of the form

$$
g_{3}(h)=\sum_{p=1}^{\infty}(-1)^{p+1} \alpha_{p} h^{2 p}
$$

and for the high values of $h$ :

$$
g_{3}(h)=h^{1 / 2}-\beta_{0}-\sum_{p=1}^{\infty} \frac{\beta_{p}}{h^{2 p-1 / 2}} .
$$

So with an accuracy better than $2.5 \times 10^{-4}$ we propose :

$$
\begin{aligned}
g_{3}(h) & \simeq 5.6464 \times 10^{-2} h^{2} \\
& -1.4759 \times 10^{-3} h^{4}+4.2747 \times 10^{-5} h^{6} \\
& -1.5351 \times 10^{-6} h^{8}+6 \times 10^{-8} h^{10}
\end{aligned}
$$

for $h \leqslant 3$

$$
\begin{aligned}
g_{3}(h) \simeq h^{1 / 2}-1.2942-\frac{\pi^{2}}{12 h^{3 / 2}}-\frac{\pi^{4}}{16 h^{7 / 2}} & \\
& -\frac{5 \pi^{6}}{32 h^{11 / 2}}
\end{aligned}
$$

for $h \geqslant 8$

- and the polynomial :

$$
\begin{array}{rl}
g_{3}(h) \simeq 0 & 64548+0.235 \delta-7.45 \times 10^{-4} \delta^{2} \\
& -2.94 \times 10^{-3} \delta^{3}+6.32 \times 10^{-4} \delta^{4} \\
& -5.22 \times 10^{-5} \delta^{5}
\end{array}
$$

for $3 \leqslant h \leqslant 8$ with $\delta=h-4$.

\section{Experimental results on the amorphous alloys $\mathrm{La}_{3} \mathrm{Al}$ and $\mathrm{La}_{3} \mathrm{Ga}$.}

The magnetoresistance of amorphous alloys $\mathrm{La}_{x} \mathrm{Al}_{1-x}$ has already been measured by $\mathrm{Lu}$ and Tsai [17] at $4.2 \mathrm{~K}$ and interpreted in terms of electron-electron interactions. However the effect of superconducting fluctuations is very important in these systems. We have measured $T_{\mathrm{c}}=3.9 \mathrm{~K}$ [18] for amorphous $\mathrm{La}_{3} \mathrm{Al}$. In order to lower the superconducting transition temperature and also the contribution of superconducting fluctuations we have introduced a few percent of praseodymium atoms in our alloys and then studied the systems $\mathrm{La}_{68} \mathrm{Pr}_{7} \mathrm{Al}_{25}$ and $\mathrm{La}_{69} \operatorname{Pr}_{6} \mathrm{Ga}_{25}$. The materials were quenched in the amorphous state by the piston-and-anvil technique at the University L. Pasteur (Strasbourg, France). Magnetoresistance measurements were performed on the one hand in a superconducting coil in Grenoble in collaboration with $O$. Laborde and on the other 
hand in a pulsed field in Toulouse. The two types of measurements gave the same results in the common field region. We have only interpreted those common results to avoid any additional contribution from normal magnetoresistance in higher fields.

In figure 1 we present the experimental curves (full lines) obtained on the alloy $\mathrm{La}_{68} \operatorname{Pr}_{7} \mathrm{Al}_{25}$. We have calculated the magnetoresistance arising from interactions in the two systems. The contribution due to spin splitting of conduction-electron energies (Eq. (21)) is proved to be negligible even if large values for the screening parameter $F(0<F<1)$, are used while the orbital contribution (Eqs. (10) and (11)) is represented by the broken lines in figure 1 .

To determine the coupling constant $g(T, H)$ we have used the values of $T_{\mathrm{c}}$ measured on the systems $\mathrm{La}_{75-x} \operatorname{Pr}_{x} \mathrm{Al}_{25}$ and $\mathrm{La}_{75-x} \operatorname{Pr}_{x} \mathrm{Ga}_{25}$ [18] $\left(T_{\mathrm{c}}=1.1 \mathrm{~K}\right.$ for $\mathrm{La}_{68} \operatorname{Pr}_{7} \mathrm{Al}_{25}$ and $T_{\mathrm{c}}=1.6 \mathrm{~K}$ for $\left.\mathrm{La}_{69} \operatorname{Pr}_{6} \mathrm{Ga}_{25}\right)$. The values of the diffusion constant were deduced from resistivity measurements $\left(D=9.4 \times 10^{-5} \mathrm{~m}^{2} / \mathrm{s}\right.$ for $\mathrm{Al}$ alloys and $D=12.9 \times 10^{-5} \mathrm{~m}^{2} / \mathrm{s}$ for $\mathrm{Ga}$ alloys).

We have then tried to fit the magnetoresistance due to weak localization and superconducting fluctuations using equation (1) and the analytic forms of the function $f_{3}(h)$. So we could give the parameters $H_{\mathrm{i}}$ and $H_{\text {so }}$ corresponding to the best fit (Fig. 2 and 3). An unresolved problem is the determination of $\beta(T, H)$ because Larkin [13] has only tabulated $\beta(T)$ as a function of $g(T)$. We know, now how to calculate $g(T, H)$, using the expression of McLean and Tsuzuki [16], but not $\beta(T, H)$. Nevertheless we have used the tabulations of Larkin, in the absence

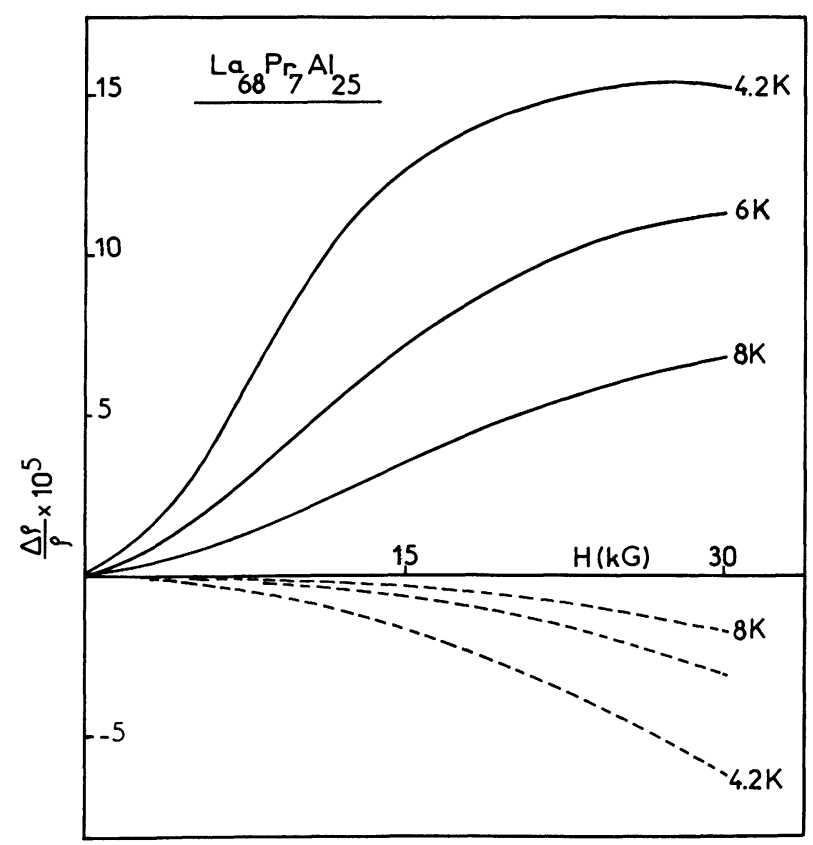

Fig. 1. - Experimental magnetoresistance (full lines) and calculated contribution arising from electron-electron interactions (broken lines) for the amorphous alloy $\mathrm{La}_{68} \mathrm{Pr}_{7} \mathrm{Al}_{25}$.

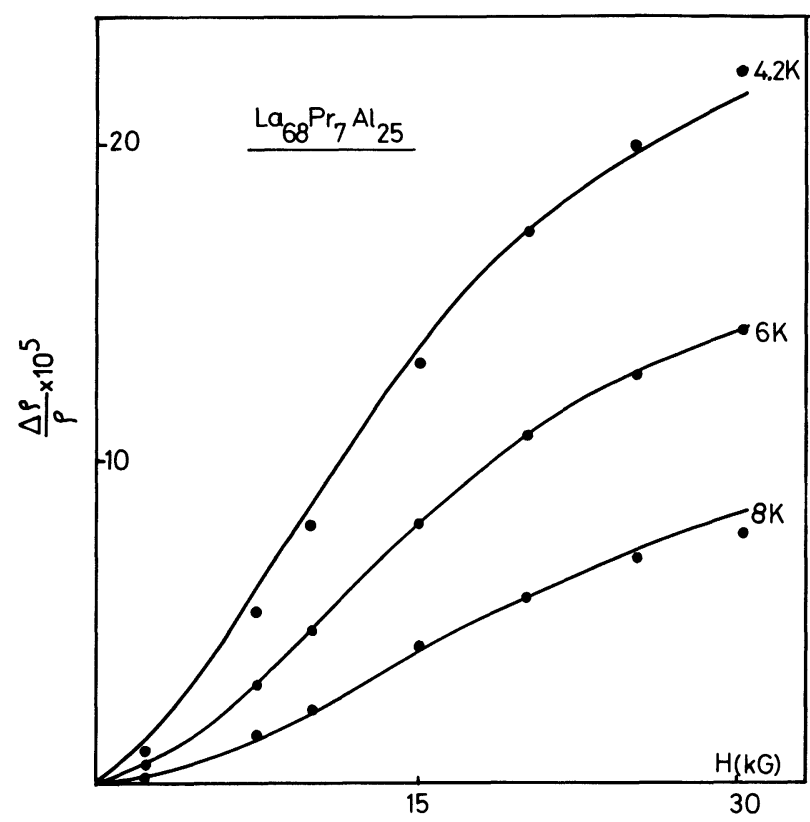

Fig. 2. - Magnetoresistance arising from localization and superconducting fluctuations for a $\mathrm{La}_{68} \mathrm{Pr}_{7} \mathrm{Al}_{25}$. The points correspond to the best fit obtained from theoretical calculations.

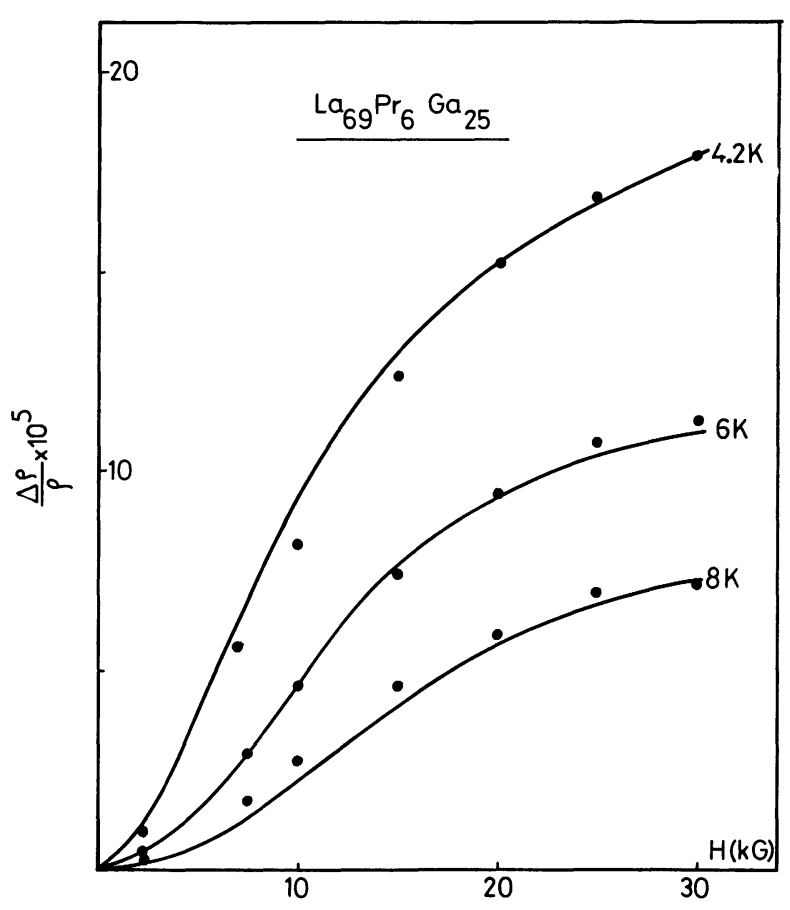

Fig. 3. - Magnetoresistance arising from localization and superconducting fluctuations for a $\mathrm{La}_{69} \operatorname{Pr}_{6} \mathrm{Ga}_{25}$. The points correspond to the best fit.

of a more comprehensive theory. In table I, we give the values of $\tau_{i}^{-1}$ and $\tau_{\text {so }}^{-1}$ deduced for our alloys. It is remarkable we found the same $\tau_{\text {so }}^{-1}$ in alloys of same concentration of lanthanum, that characteristic of a strong spin-orbit scattering on lanthanum sites. 
Table I. - Values of $\tau_{\mathrm{i}}^{-1}$ as a function of the temperature and $\tau_{\mathrm{so}}^{-1}$ for the two studied alloys.

\begin{tabular}{clll} 
& & $\mathrm{La}_{68} \mathrm{Pr}_{7} \mathrm{Al}_{25}$ & $\mathrm{La}_{69} \mathrm{Pr}_{6} \mathrm{Ga}_{25}$ \\
\hline & $T=4.2 \mathrm{~K}$ & $2.41 \times 10^{11}$ & $2.90 \times 10^{11}$ \\
$\tau_{\mathrm{i}}^{-1}$ & $T=6 \mathrm{~K}$ & $2.94 \times 10^{11}$ & $3.54 \times 10^{11}$ \\
$\left(\mathrm{~s}^{-1}\right)$ & $T=8 \mathrm{~K}$ & $4.01 \times 10^{11}$ & $4.17 \times 10^{11}$ \\
\hline$\tau_{\text {so }}^{-1}\left(\mathrm{~s}^{-1}\right)$ & & $3.33 \times 10^{11}$ & $3.36 \times 10^{11}$ \\
\hline
\end{tabular}

The values of $\tau_{\mathbf{i}}^{-1}$ can be considered as a good approximation of the inelastic relaxation times because, although, the superconducting fluctuations were predominant in these systems (higher by a factor 4 than localization effects for $\mathrm{La}_{68} \mathrm{Pr}_{7} \mathrm{Al}_{25}$ at $T=4.2 \mathrm{~K}$ and $H=3 \mathrm{~T}$ ), their contribution also includes the parameter $H_{\mathrm{i}}$. We could not clearly establish a temperature dependence of the form $\tau_{i}^{-1} \simeq T^{\alpha}$ in the case of the alloy containing aluminium while for gallium alloy we find a value for $\alpha$ about 0.75 . The temperature range is however too small to discuss this variation in detail.

\section{Conclusion.}

In the present paper we have reviewed the anormal magnetoresistance effects observed in metallic disordered systems and given analytic expressions in order to simplify the interpretation of experimental results in any range of magnetic field values. So we could deduce with a reasonable precision both inelastic and spin-orbit relaxation times in the case of amorphous $\mathrm{La}_{3} \mathrm{Al}$ and $\mathrm{La}_{3} \mathrm{Ga}$ containing praseodymium. A good agreement is obtained between theory and experiments. However discrepancies appear when measuring the magnetoresistance up to higher fields. We shall discuss these problems in a further publication.

\section{References}

[1] Anderson, P. W., Phys. Rev. 109 (1958) 1492.

[2] Bergmann, G., Phys. Rev. Lett. 43 (1979) 1357.

[3] Van Haesendonck, C., Van Den Dries, L., BruynSRAede and Deutscher, G., Phys. Rev. B 25 (1982) 5090 .

[4] Abraham, D. and Rosenbaum, R., J. Phys. C 17 (1984) 2627.

[5] Chui, T., Lindenfeld, P., McLean, W. L. and Mui, K., Phys. Rev. Lett. 47 (1981) 1617.

[6] Werner, T. R., BanerJee, I., Yang, Q. S., Falco, C. M. and Schuller, I. K., Phys. Rev. B 26 (1982) 2224.

[7] Dynes, R. C., Geballe, T. H., Hull, G. W. and Garno, J. P., Phys. Rev. B 27 (1983) 1588.

[8] Bieri, J. B., Fert, A., Creuzet, G. and Ousset, J. C., Solid State Commun. 49 (1984) 849.

[9] Cochrane, R. N. and Strom-Olsen, J. O., Phys. Rev. B 29 (1984) 1080.
[10] Howson, M. A. and Greig, D., J. Phys. F 13 (1983) L155.

[11] Kawabata, A., Solid State Commun. 34 (1980) 431.

[12] Alt'shuler, B. L., Aronov, A. G., Larkin, A. I. and KhMel'NitskiI, D. E., Sov. Phys. JETP 54 (1981) 411.

[13] LARKIN, A. I., JETP Lett. 31 (1980) 219.

[14] Lee, P. A. and Ramakrishnan, T. V., Phys. Rev. B 26 (1982) 4009.

[15] Gershenson, M. E., Gubankov, V. N. and Zhuralev, Y. E., Solid State Commun. 45 (1983) 87.

[16] Mclean, W. L. and Tsuzuki, T., Phys. Rev. B 29 (1984) 503.

[17] Lu, F. C. and TsaI, C. L., Magnetism and Magnetic Materials, PitTSBURG (1983).

[18] Ousset, J. C., Cantaloup, S., Durand, J. and AskeNAZY, S., to be published. 